\title{
Influências das condições de trabalho do pai sobre o relacionamento pai-filho
}

\author{
Fabiana Cia ${ }^{1}$ \\ Elizabeth Joan Barham
}

\begin{abstract}
Resumo
O presente estudo teve por objetivo identificar as condições de trabalho que influenciam no envolvimento do pai com seu filho. Participaram deste estudo 58 pais que tinham um filho na $5 \underline{a}$ ou 6a série do Ensino Fundamental. Os pais preencheram o questionário "Avaliação das condições de trabalho e do envolvimento do pai com seu filho versão paterna". As medidas das condições de trabalho apresentaram correlações significativas com a satisfação do pai em relação ao seu desempenho familiar. Esta escala, por sua vez, estava positivamente correlacionada com as medidas do envolvimento do pai com seu filho. Assim, a relação entre as condições de trabalho e o envolvimento do pai com seu filho foi mediada pela satisfação do pai com seu desempenho familiar. Tais resultados demonstram a necessidade de identificar e alterar as normas e condições de trabalho que restringem as oportunidades para os pais participarem das rotinas familiares.

Palavras-chave: Relacionamento entre pai e filho; Condições de trabalho; Pai.
\end{abstract}

\section{Influences father's work conditions on father-child relationship}

\begin{abstract}
The aim of this study was to identify the work conditions that influence father-child relationship. A total of 58 fathers, whose child was at fifth or sixth grade, participated in this study. The fathers completed the questionnaire "Evaluation of work conditions and involvement of the father with his child - Paternal version". The measures of work conditions presented significant correlations with father's satisfaction concerning his family performance. This scale, in turn, presented correlations with the measures of father-child involvement. Therefore, the relation between work conditions and father-child involvement was mediated by the satisfaction of the father with his family performance. These results indicate the need to identify and alter the work rules and conditions, which restrict fathers' opportunities to participate in their family routines.
\end{abstract}

Keywords: Father-child relationship; Work conditions; Father.

\section{Introdução}

\section{Equilibrio entre trabalho e familia}

A partir da década de 1970, como resultado da revolução feminista, está aumentando o número de mulheres que exercem atividades remuneradas. Como resultado, surgiu a necessidade de contar com um envolvimento maior por parte dos homens para com as responsabilidades familiares (Brandth \& Kvande, 2002; Rodrigues, Assmar \& Jablonski, 2002; Tiedje, 2004).

Esta nova realidade social, em que a maioria das famílias combina trabalho remunerado e familiar, alterou as relações de poder entre homens e mulheres, havendo uma transformação nos papéis sociais ligados ao gênero no que diz respeito à participação em trabalho fora e dentro de casa (Bertolini, 2002; Lamb, 1997; Lewis \& Dessen, 1999). Muitos homens, cujas mulheres trabalham fora, enfrentam grandes dificuldades em apresentar um desempenho familiar satisfatório (como por exemplo, dividir tarefas domésticas e educar os filhos) e cumprir normas de trabalho. Além disso, precisam reavaliar seu próprio papel enquanto provedor familiar, uma vez que passaram a dividir essa posição, tão valorizada, com suas mulheres. Sendo assim, o papel do homem se encontra numa fase de transição social, sendo redefinido constantemente (Cabrera, Tames-LeMonda, Bradley, Hofferth \& Lamb, 2000; Coley, 2001; Diniz, 1999; Tiedje, 2004).

Estudos realizados com homens e mulheres britânicos, que trabalhavam em tempo integral, demonstraram que os conflitos entre trabalho e família surgem quando ocorre sobreposição das demandas familiares e profissionais (Cooper \& Lewis, 2000). Os conflitos podem estar relacionados ao tempo (invasão de horários) ou à sobrecarga (cansaço), com parte destes tendo origem no trabalho remunerado e a outra parte na família (Gottlieb, Kelloway \& Barham, 1998). Os relacionados ao tempo, envolvem a existência de demandas das duas esferas simultaneamente - trabalho e

\footnotetext{
${ }^{1}$ Endereço para correspondência:

Universidade Federal de São Carlos - Centro de Educação e Ciências Humanas, Departamento de Psicologia - LABOR

Rodovia Washington Luís - Km 235 - São Carlos-SP

E-mail: fabianacia@hotmail.com
} 
família. Os conflitos relacionados à sobrecarga referem-se ao excesso de preocupações e responsabilidades em uma esfera (família ou trabalho), afetando a participação e desempenho na outra. A sobreposição das demandas de cada esfera acaba exigindo que os indivíduos escolham entre os dois papéis, tendo que optar entre as necessidades familiares e as solicitações ou demandas do trabalho. Geralmente, o processo de escolha entre as demandas familiares ou de trabalho acaba acarretando custos, tanto para a esfera familiar quanto para a profissional.

Warren e Johnson (1995) notaram que, quando homens exercem atividade remunerada fora do lar, a demanda de tempo e energia para conciliar as obrigações familiares e profissionais é um fator de estresse que pode contribuir para o aumento da ansiedade e sentimento de culpa decorrente da pouca participação na educação dos filhos.

\section{A figura paterna e o desenvolvimento infantil}

A privação paterna ou uma interação inadequada com o pai é considerada um fator de risco para o desenvolvimento infantil (Dunn, 2004; Patterson, Mockford \& Stewart-Brow, 2005; Ramchandani \& McConachie, 2005). O ideal seria a criança se desenvolver ao lado de ambos os pais, pois cada um contribui positivamente em alguns aspectos no desenvolvimento infantil (Black, Dubowitz \& Starr, 1999; Dessen \& Costa, 2005; Flouri \& Buchanan, 2003).

As pesquisas têm mostrado, de modo geral, que o pai é importante para participar dos cuidados com os filhos e das atividades domésticas, para ajudar a suprir as necessidades financeiras da família (com rendas familiares maiores, permitindo acesso a uma alimentação de melhor qualidade, maiores oportunidades de lazer e maiores cuidados para com a saúde) e para motivar seus filhos na escola (Black e colaboradores, 1999; Coley, 1998; Dubowitz e colaboradores, 2001; Dunn, 2004; Hill \& Taylor, 2004). Ao mesmo tempo, tais comportamentos por parte dos pais contribuiriam indiretamente para o melhor relacionamento mãe/filho, pois as mães estariam menos sobrecarregadas (Lamb, 1997).

Amato e Gilbreth (1999), Engle e Breaux (1998) ainda completam que o relacionamento seguro com o pai pode contribuir para o bem-estar emocional e compensar um mau relacionamento com a mãe, quando existe. No entanto, apesar de muitos pais quererem se envolver com seus filhos, podem existir condições de trabalho que dificultam o estabelecimento de um relacionamento adequado entre o pai e seu filho (Diniz, 1999; Gottlieb e colaboradores; 1998; Robbins, 1998). Sendo assim, os objetivos deste estudo foram:

1. Descrever as condições de trabalho dos pais e o relacionamento entre pais e filhos e
2. Identificar as condições de trabalho que influenciam o envolvimento do pai com seu filho.

\section{Método}

\section{Participantes}

Participaram deste estudo 58 pais (36 que trabalhavam no turno diurno e 22 no noturno), com média de idade de 39 anos, variando entre 31 e 61 anos, sendo a maioria de classe socioeconômica baixa. Em relação ao grau de escolaridade, $41,4 \%$ dos pais possuíam $2^{\circ}$ grau completo. Estes eram trabalhadores de diversas empresas do setor industrial de um município do interior do estado de São Paulo e trabalhavam, em média, 45 horas semanais. Todos os pais tinham um filho com idade média de 12 anos, variando entre 10 e 14 anos. Dessas crianças, 30 foram do sexo feminino e 28 do sexo masculino, e exatamente a metade delas estava na $5 \underline{a}$ série e metade na 6 série do Ensino Fundamental.

\section{Local da coleta de dados}

A coleta de dados com os pais ocorreu em uma escola pública, mantida por indústrias, localizada em uma cidade de médio porte no interior do estado de São Paulo.

\section{Medidas avaliativas - questionário para os pais}

Os pais preencheram o instrumento "Avaliação das condições de trabalho e do envolvimento do pai com seu filho - versão paterna" construído pelas pesquisadoras com base em instrumentos já existentes (Bolsoni-Silva, Del Prette \& Del Prette, 2000; Canadian Aging Research Network, 1995). Este instrumento está dividido em dois tópicos:

\section{Condições de trabalho}

- Escala de ambiente interpessoal de trabalho: uma escala tipo Likert de 10 itens (por exemplo: você pode falar sobre assuntos pessoais com seus colegas de trabalho; seu superior respeita suas responsabilidades pessoais/familiares) para o respondente avaliar o seu ambiente de trabalho, com relação ao supervisor, a ele mesmo e aos colegas, tendo cada item pontuado entre 1 "discordo totalmente" e 5 "concordo totalmente";

- Escala de satisfação com o trabalho: uma escala tipo Likert de 15 itens (por exemplo: quanto você se sente bem com aquilo que faz; a carreira que você seguiu) que avalia o grau de satisfação do respondente quanto às condições de trabalho, com cada item pontuado entre 1 "muito insatisfeito" e 5 "muito satisfeito";

- Escala de problemas com o desempenho no trabalho: uma escala tipo Likert de 11 itens (por exemplo: não pude participar de algumas reuniões; não pude aceitar 
projetos/horas extras), que avalia a influência com a qual as demandas pessoais e familiares resultam em falhas no desempenho profissional do respondente, com cada item pontuado entre 0 "nunca" e 365 "todo dia";

- Escala de atividades pessoais: uma escala tipo Likert de nove itens (por exemplo: atividade física; cuidar da aparência pessoal), que avalia a satisfação do respondente em relação à disponibilidade de tempo fora do seu expediente de trabalho, para realizar atividades pessoais, com cada item pontuado entre 1 "muito insatisfeito" e 5 "muito satisfeito";

- Escala de estresse: uma escala tipo Likert de 11 itens (por exemplo: sentiu-se esgotado e cansado; teve dificuldade para se concentrar), que avalia a freqüência com a qual o respondente sente-se estressado, de forma geral, com cada item pontuado entre 1 "nunca" e 5 "sempre";

- Escala de adequação do desempenho no papel familiar: uma escala tipo Likert de oito itens (por exemplo: sinto que eu estou tão próximo do meu filho como eu gostaria de estar; eu não estou colaborando com minha família como gostaria de estar), que avalia a percepção do respondente em relação à adequação do seu desempenho no papel familiar, com cada item pontuado entre 1 "discordo totalmente" e 5 "concordo totalmente".

\section{Relacionamento entre pai e filho}

- Escala de comunicação (verbal e não verbal) iniciada pelo pai, para com seu filho: uma escala tipo Likert de 12 itens (por exemplo: você dá carinho a seu filho; você elogia seu filho), com cada item pontuado entre 0 "nunca" e 365 "uma vez por dia";

- Escala de comunicação (verbal e não-verbal) iniciada pelo filho, para com seu pai: uma escala tipo Likert de 10 itens (por exemplo: seu filho conta as coisas boas ou ruins ocorridas com ele em relação à escola; seu filho desafia suas regras), com cada item pontuado entre 0 "nunca" e 365 "uma vez por dia";

- Escala de participação do pai nos cuidados com o filho: uma escala tipo Likert de 16 itens (por exemplo: controlar o círculo de amizades de seu filho; punir seu filho por comportamento inadequado), com cada item pontuado entre 1 "pouca participação" e 5 "muita participação";

- Escala de participação do pai na realização de atividades escolares, culturais e de lazer com o filho: uma escala tipo Likert de 19 itens (por exemplo: incentivar seu filho a ler; brincar com seu filho), com cada item pontuado entre 0 "nunca" e 365 "todos os dias".

\section{Procedimento de coleta de dados}

O primeiro contato com os pais se deu por meio de uma reunião, em que a primeira autora explicou os objetivos do estudo e a forma como ocorreria a coleta de dados. Em seguida, aos pais que tiveram interesse em participar do estudo foi entregue o questionário, explicado cada item deste instrumento e respondidas às dúvidas apontadas pelos pais. Estes também receberam o Termo de Consentimento Livre e Esclarecido para sua participação.

\section{Procedimento de análise de dados}

Os dados quantitativos obtidos com o questionário preenchido pelos pais foram analisados estatisticamente segundo medidas de tendência central e dispersão. Para analisar os itens que poderiam compor uma escala e para avaliar a fidedignidade das medidas (Cozby, 2002), verificou-se a consistência interna calculando o alfa de Cronbach da escala como um todo. Para investigar a relação entre as condições de trabalho e o relacionamento pai-filho, foi utilizado o Teste de Correlação de Pearson.

\section{Resultados}

\section{Condicões de trabalho do pai}

Os dados da Tabela 1 mostram as condições de trabalho do pai.

Tabela 1 - Condições de trabalho do pai

\begin{tabular}{|c|c|c|c|}
\hline Escala & média & $\mathrm{DP}$ & $\begin{array}{c}\text { Alfa de } \\
\text { Cronbach }\end{array}$ \\
\hline $\begin{array}{l}\text { Ambiente interpessoal de } \\
\text { trabalho } \\
1=\text { discordo totalmente a } \\
5=\text { concordo totalmente }\end{array}$ & 3,31 & 0,56 & 0,70 \\
\hline $\begin{array}{l}\text { Satisfação com o trabalho } \\
1=\text { muito insatisfeito a } \\
5=\text { muito satisfeito }\end{array}$ & 3,45 & 0,57 & 0,87 \\
\hline $\begin{array}{l}\text { Problemas com o } \\
\text { desempenho no trabalho } \\
0=\text { nunca a } 365=\text { todos } \\
\text { os dias }\end{array}$ & 22,37 & 37,79 & 0,77 \\
\hline $\begin{array}{l}\text { Satisfação com a realização } \\
\text { de atividades pessoais } \\
1=\text { muito insatisfeito a } \\
5=\text { muito satisfeito }\end{array}$ & 3,14 & 0,67 & 0,83 \\
\hline $\begin{array}{l}\text { Estresse } \\
1=\text { nunca, sem estresse a } \\
5=\text { sempre, estresse } \\
\text { intenso }\end{array}$ & 2,83 & 0,52 & 0,75 \\
\hline $\begin{array}{l}\text { Adequação do desempenho } \\
\text { no papel familiar } \\
1=\text { discordo totalmente a } \\
5=\text { concordo totalmente }\end{array}$ & 3,23 & 0,72 & 0,77 \\
\hline
\end{tabular}


Os pais apresentaram pontuações medianas em relação ao ambiente interpessoal de trabalho, satisfação com o trabalho, satisfação com realização de atividades pessoais, estresse e adequação do desempenho no papel familiar e mostraram baixa freqüência (aproximadamente dois problemas enfrentados a cada mês de trabalho) de problemas com o desempenho no trabalho. A

Tabela 2 apresenta as correlações existentes entre as medidas das condições de trabalho.

Tabela 2 - Correlação entre as medidas das condições de trabalho

\begin{tabular}{lccccc}
\hline & \multicolumn{5}{c}{ Teste de Pearson r } \\
& 1 & 2 & 3 & 4 & 5 \\
\hline 1- ambiente interpessoal de trabalho & - & & & & \\
2- satisfação com o trabalho & $0,587 * * *$ & - & & & \\
3- problemas com o desempenho no trabalho & $-0,044$ & $-0,056$ & - & - \\
4- atividades pessoais & $0,297 *$ & $0,453^{* * *}$ & $-0,133$ & $-0,443^{* *}$ & - \\
5- estresse & $-0,224+$ & $-0,347^{* *}$ & $0,227+$ & $-0,489^{* * *}$ & $-0,345^{* *}$ \\
6- adequação do desempenho no papel familiar & $0,319^{*}$ & $0,377^{* *}$ & $-0,261+$ & &
\end{tabular}

Quanto menor a satisfação com a realização de atividades pessoais (deixar de se cuidar), maior o estresse dos respondentes. A freqüência da realização das atividades pessoais também se correlacionou positivamente com a adequação do desempenho no papel familiar, com a qualidade do ambiente interpessoal de trabalho e com a satisfação com o trabalho. Além disso, quanto maior o estresse dos pais menor a qualidade do ambiente interpessoal de trabalho, a satisfação com o trabalho e a adequação do desempenho no papel familiar. $O$ estresse se correlacionou positivamente com os problemas com o desempenho no trabalho.

Em relação à adequação do desempenho no papel familiar, pôde-se verificar que se correlacionou positivamente com a qualidade do ambiente interpessoal de trabalho e com a satisfação com o trabalho, e negativamente com os problemas com o desempenho no trabalho.

Tabela 3-Qualidade do relacionamento entre pai e filho

\begin{tabular}{|c|c|c|c|}
\hline Escala & Média & DP & $\begin{array}{l}\text { Alfa de } \\
\text { Cronbach }\end{array}$ \\
\hline $\begin{array}{l}\text { Comunicação (verbal e não verbal) iniciada pelo pai, para com seu filho } \\
0=\text { nunca a } 365=\text { todos os dias }\end{array}$ & 223,8 & 98,7 & 0,88 \\
\hline $\begin{array}{l}\text { Comunicação (verbal e não verbal) iniciada pelo filho, para com seu pai } \\
0=\text { nunca a } 365=\text { todos os dias }\end{array}$ & 253,3 & 100,8 & 0,88 \\
\hline $\begin{array}{l}\text { Participação do pai nos cuidados com o filho } \\
1=\text { pouca participação a } 5=\text { muita participação }\end{array}$ & 4,2 & 0,80 & 0,87 \\
\hline $\begin{array}{l}\text { Participação do pai nas atividades escolares, culturais e de lazer com o filho } \\
0=\text { nunca a } 365=\text { todos os dias }\end{array}$ & 259,3 & 87,7 & 0,91 \\
\hline
\end{tabular}

\section{Relacionamento entre pai e filho}

Os dados da Tabela 3 mostram a qualidade do relacionamento entre pai e filho.

Os pais desta amostra apresentaram alta freqüência (aproximadamente uma vez por dia) em relação aos quatro aspectos avaliados do relacionamento entre pai e filho. Sendo assim, em média, a maioria dos pais relatou que tanto eles, quanto seus filhos, usavam todas as formas de comunicação incluídas neste estudo, quase todos os dias.
Esses pais também apontaram alta participação nos cuidados com os filhos e nas atividades escolares, culturais e de lazer com os filhos.

Correlação entre as condições de trabalho do pai e o relacionamento entre pai e filho

$\mathrm{Na}$ Tabela 4 encontram-se as correlações entre as condições de trabalho do pai e o relacionamento entre pai e filho. 
Tabela 4 - Correlação entre as condições de trabalho do pai e o relacionamento entre pai e filho

\begin{tabular}{|c|c|c|c|c|}
\hline \multicolumn{5}{|c|}{ Teste de Pearson $\mathrm{r}$} \\
\hline & $\begin{array}{l}\text { Comunicações } \\
\text { iniciadas pelo pai, } \\
\text { para com seu filho }\end{array}$ & $\begin{array}{c}\text { Comunicações } \\
\text { iniciadas pelo filho, } \\
\text { para com seu pai }\end{array}$ & $\begin{array}{l}\text { Participação do } \\
\text { pai nos cuidados } \\
\text { com o filho }\end{array}$ & $\begin{array}{c}\text { Participação do pai nas } \\
\text { atividades escolares, } \\
\text { culturais e de lazer com o } \\
\text { filho }\end{array}$ \\
\hline $\begin{array}{l}\text { 1- ambiente } \\
\text { interpessoal de } \\
\text { trabalho }\end{array}$ & 0,169 & 0,171 & 0,084 & 0,191 \\
\hline $\begin{array}{l}\text { 2- satisfação com o } \\
\text { trabalho }\end{array}$ & 0,128 & 0,003 & $-0,087$ & 0,065 \\
\hline $\begin{array}{l}\text { 3- problemas com o } \\
\text { desempenho no } \\
\text { trabalho }\end{array}$ & 0,106 & 0,164 & $-0,052$ & 0,051 \\
\hline 4- atividades pessoais & 0,144 & 0,011 & 0,012 & 0,168 \\
\hline 5- estresse & $-0,104$ & $-0,115$ & $-0,104$ & $-0,089$ \\
\hline $\begin{array}{l}\text { 6- adequação do } \\
\text { desempenho no papel } \\
\text { familiar }\end{array}$ & $0,345^{* *}$ & $0,302 *$ & $0,312^{*}$ & $0,294 *$ \\
\hline
\end{tabular}

Nota: ${ }^{*} p<0,05 ; * * p<0,01$.

Apareceram correlações significativas e positivas entre as quatro medidas do relacionamento entre pai e filho e a adequação do desempenho no papel familiar, apenas.

\section{Discussão}

Considerando as condições de trabalho destes pais, pode-se supor que um ambiente interpessoal de trabalho que pouco leva em consideração as demandas familiares dos respondentes tenha contribuído diretamente para a menor adequação do desempenho no papel familiar, por parte dos pais. Pois, em um ambiente interpessoal de trabalho pouco adequado, há pouca oportunidade para os pais conversarem com colegas sobre seus problemas familiares, recebendo assim, menos apoio psicológico e prático na resolução das suas dificuldades, o que está relacionado com o aumento do nível de estresse e menor satisfação quanto à realização de atividades pessoais dos respondentes (Figueroa, Schufer, Muiñoes, Mano \& Corea, 2001; Tamayo \& Trócolli, 2002).

A freqüência de satisfação quanto à realização de atividades pessoais estava positivamente correlacionada com a adequação do desempenho no papel familiar. Ou seja, os pais estavam se dedicando da mesma forma nos papéis profissionais, familiares e pessoais (por exemplo, realizar atividades físicas e descansar adequadamente). As correlações negativas entre estresse e satisfação no trabalho e entre estresse e adequação do desempenho no papel familiar dão apoio para esta interpretação, uma vez que, os pais estavam com um índice de estresse mediano, o que reflete na satisfação mediana quanto ao seu trabalho e quanto ao seu desempenho no papel familiar (Chiavenato, 1997; Robbins, 1998; Siqueira, 2002).

No entanto, o estresse pode prejudicar o relacionamento entre pai e filho, pois os pais passam a não ter tempo ou energia/vontade para realizar atividades com seus filhos (Brandth \& Kvande, 2002; Lewis \& Dessen, 1999). O nível de estresse mediano apresentado pelos pais desta amostra pode estar influenciando no fato deles não estarem tão próximos dos seus filhos quanto gostariam de estar, como foi apontado por estes pais. Conger e colaboradores (2002), Goldberg, Clarke-Stewart, Rice e Dellis (2002) apontam que o estresse no trabalho é precursor de conflitos parentais, agindo diretamente no relacionamento de ambos os pais com seus filhos, provocando uma falta de responsabilidade, comportamentos controladores, rejeição e cordialidade. É interessante ressaltar que o estresse desses pais também pode estar relacionado ao baixo nível socioeconômico, que, por sua vez, influencia diretamente no relacionamento familiar e no trabalho (Conger e colaboradores, 2002).

Nota-se que, quanto maior a satisfação no trabalho, maior a auto-avaliação dos pais sobre a adequação do desempenho no papel familiar. Lembrase que o trabalho é uma fonte muito importante de auto-estima, e que pessoas mais satisfeitas com seu 
trabalho têm melhor saúde mental (Borges \& Alves, 2001; Robbins, 1998; Siqueira, 2002), possuindo condições psicológicas melhores para se ter um adequado desempenho familiar. No entanto, a maioria dos respondentes deste estudo não estava muito satisfeita com sua condição de trabalho, o que pode explicar a satisfação mediana com o desempenho no papel familiar.

No relacionamento entre pai e filho, de modo geral, os pais indicaram que mantiveram diversas formas de comunicações diariamente com seus filhos, avaliaram sua participação quanto aos cuidados dos filhos como alta e apontaram que participavam das atividades escolares, culturais e de lazer dos filhos com alta freqüência. Este relacionamento significativo entre pai e filho é precursor de um bom desenvolvimento infantil, podendo-se destacar: melhor desenvolvimento social (Bolsoni-Silva e colaboradores, 2000; Flouri, 2005; Patterson e colaboradores, 2005), melhor autoconceito (Dekovic \& Meuis, 1997; Verschueren \& Marcoen, 1999), melhor desenvolvimento cognitivo (Black e colaboradores, 1999; Dubowitz e colaboradores, 2001) e melhor performance escolar (Flouri \& Buchanan, 2003; Hill \& Taylor, 2004). Tais fatores, por sua vez, auxiliam no melhor ajustamento psicossocial dos filhos na fase adulta (Flouri \& Buchanan, 2003).

A Figura 1 mostra as correlações existentes entre as condições de trabalho do pai e o relacionamento entre pai e filho.

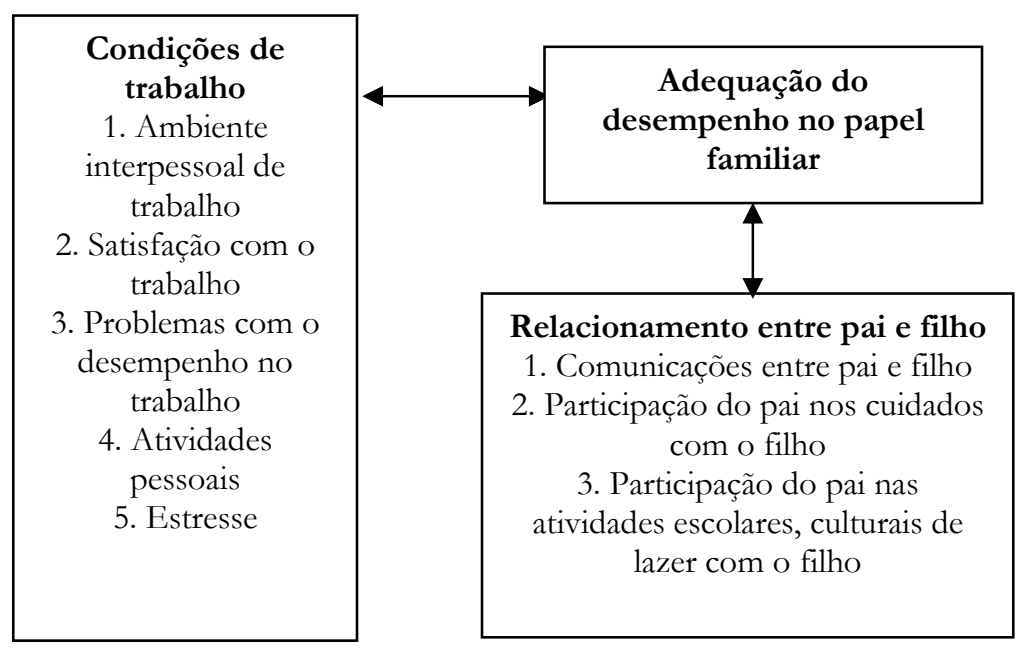

Figura 1 - Correlação entre condições de trabalho do pai e o relacionamento entre pai e filho

As condições de trabalho dos pais não apresentaram correlações diretas com o relacionamento entre pai e filho, mas apresentaram correlações significativas com a adequação do desempenho no papel familiar que, por sua vez, apresentou correlação significativa e positiva com o relacionamento entre pai e filho, concordando com os achados das pesquisas de Conger e colaboradores (2002), Cooper e Lewis (2000), Gottlieb e colaboradores (1998). Como as condições de trabalho foram apontadas pelos pais como medianas, a qualidade do relacionamento com os filhos poderia ser maximizada se houvesse melhores condições de trabalho.

\section{Considerações finais}

Este estudo levantou questões acerca das condições de trabalho que possam influenciar o envolvimento do pai com seu filho. Pode-se concluir que os aspectos de trabalho influenciavam indiretamente no relacionamento entre pai e filho e diretamente na satisfação do pai quanto ao seu desempenho no papel familiar. Estes resultados são sugestivos da necessidade de iniciativas para mudar ou amenizar o impacto da rotina de trabalho, principalmente para funcionários que são pais. Os pais se beneficiariam se as empresas oferecessem melhores condições de trabalho, como um ambiente interpessoal 
de trabalho mais favorável, flexibilidade de horários, horários de trabalho parciais ou trabalho em casa para os trabalhadores terem maiores oportunidades para resolver os problemas pessoais e familiares, como já existe, mesmo que informalmente, com as mulheres que trabalham e são mães.

Este estudo trouxe contribuição quanto à elaboração e aplicação de um instrumento de coleta de dados direcionado aos homens, que aborda as condições do trabalho e a qualidade do relacionamento entre pai e filho. Dessa forma, este instrumento pode ser utilizado em outros estudos de levantamento e de pesquisa-intervenção. Sugere-se que outros estudos desta ordem sejam realizados, utilizando-se maior número de participantes. Adicionalmente, estudos longitudinais seriam aconselháveis para monitorar a influência das condições de trabalho do pai sobre o relacionamento entre pai e filho, ao longo do desenvolvimento da criança.

\section{Referências}

Amato, P. R. \& Gilbreth, J. G. (1999). Nonresident fathers and children's well-being: A meta-analyses. Journal of Marriage and the Family, 61, 557-573.

Bertolini, L. B. A. (2002). Funções paternas, maternas e conjugais na sociedade ocidental. Em A. L. B. Bertolini (Org.). Relações entre o trabalho da mulher e a dinâmica familiar (pp. 27-31). São Paulo: Vetor.

Black, M. M., Dubowitz, H. \& Starr, R. H. (1999). African American fathers in low income, urban families: Development, behavior, and home environment of their three-year-old children. Child Development, 70(4), 967-978.

Bolsoni-Silva, A. T. B., Del Prette, A. \& Del Prette, Z. A. P. (2000). Relacionamento pais-filhos: um programa de desenvolvimento interpessoal em grupo. Psicologia Escolar e Educacional, 3(3), 203-215.

Borges, L. O. \& Alves, A. (2001). A mensuração da motivação e do significado do trabalho. Estudos de Psicologia, 6(2), 177-194.

Brandth, B. \& Kvande, E. (2002). Reflexive fathers: Negotiating parental leave and working life. Gender, Work and Organization, 9(2), 186-203.

Cabrera, N. J., Tames-LeMonda, C., Bradley, R. H., Hofferth, S. \& Lamb, M. E. (2000). Fatherhood in the twenty first century. Child Development, 71(1), 127136.
Canadian Aging Research Network (1995). Work and family: The survey findings for the work and eldercare research group. Guelph, ON: Autor.

Chiavenato, I. (1997). Recursos Humanos. São Paulo: Atlas.

Coley, R. L. (1998). Children's socialization experiences and functioning in single-mother households: The importance of fathers and other men. Child Development, 9(1), 219-230.

Coley, R. L. (2001). (In)visible men: Emerging research on low-income, unmarried, and minority fathers. American Psychologist, 56(9), 743-753.

Conger, R. D., McLoyd, V., Wallace, L. E., Sun, Y., Simons, R. L. \& Brody, G. H. (2002). Economic pressure in African American families. Developmental Psychology, 38(2), 179-193.

Cooper, C. L. \& Lewis, S. (2000). E agora, trabalho on família? São Paulo: Tâmisa.

Cozby, P. C. (2002). Métodos de pesquisa em ciências do comportamento. São Paulo: Atlas.

Dekovic, M. \& Meuis, W. (1997). Peer relations in adolescence: Effects so parenting and adolescents' self-concept. Journal of Adolescence, 97(20), 1163-1176.

Dessen, M. A. \& Costa, A. L. (2005). A ciência do desenvolvimento humano. Porto Alegre: Artmed.

Diniz, G. (1999). Homens e mulheres frente à interação casamento trabalho: aspectos da realidade brasileira. Em T. Féres-Carneiro (Org.). Casal e família: entre a tradição e a transformação. (pp. 31-54). Rio de Janeiro: NAU.

Dubowitz, H., Black, M. M., Cox, C. E., Kerr, M. A., Litrownik, A. J., Radhakrishna, A., English, D. J., Schneider, M. W. \& Runyan, D. K. (2001). Father involvement and children's functioning at age 6 years: A multisite study. Child Maltreatment, 6(4), 300309.

Dunn, J. (2004). Annotation: Children's relationships with their nonresident father. Journal of Child Psychology and Psychiatry, 45(4), 659-671.

Engle, P. L. \& Breaux, C. (1998). Fathers' involvement with children: Perspectives from developing countries. Social Policy Report: Society for Research in Child Development, 12(1), 1-23.

Figueroa, N. L., Schufer, M., Muiñoes, R., Mano, C. \& Corea, E. A. (2001). Um instrumento para a avaliação de estressores psicossociais no contexto do emprego. Psicologia: Reflexão e Crítica, 14(3), 635-659. 
Flouri, E. (2005). Father's involvement and psychological adjustment in Indian and White British secondary school age children. Child and Adolescent Mental Health, 10(1), 32-40.

Flouri, E. \& Buchanan, A. (2003). The role of involvement in children's later mental health. Journal of Adolescence, 26, 63-78.

Goldberg, W. A., Clarke-Stewart, K. A., Rice, J. A. \& Dellis, E. (2002). Emotional energy as an explanatory construct for fathers' engagement with their infants. Parenting: Science and Practice, 2(4), 379-408.

Gottlieb, B. H., Kelloway, E. K. \& Barham, E. J. (1998). Flexible work arrangements: Managing the work-family boundary. Chichester, Inglaterra: John Wiley \& Sons.

Hill, N. E. \& Taylor, L. C. (2004). Parental school involvement and children's academic achievement. Current Directions in Psychological Science, 13(4), 161-164.

Lamb, M. E. (1997). Fathers and child development: An introductory overview and guide. Em M. E. Lamb (Org.). The role of the father in child developmental (pp. 118). New York: John Wiley \& Sons.

Lewis, C. \& Dessen, M. A. (1999). O pai no contexto familiar. Psicologia: Teoria e Pesquisa, 15(1), 9-16.

Patterson, J., Mockford, C. \& Stewart-Brow, S. (2005). Parents' perceptions of the value of the Websterstratton Parenting Programme: A qualitative study of a general practice based iniciative. Child: Care, Health and Development, 31(1), 53-64.
Ramchandani, P. \& McConachie, H. (2005). Mother, father and their children's health. Child: Care, Health and Development, 31(1), 5-6.

Robbins, S. P. (1998). Comportamento organizacional. Rio de Janeiro: Prentice Hall.

Rodrigues, A., Assmar, E. M. L. \& Jablonski, B. (2002). Psicologia social. Petrópolis: Vozes.

Siqueira, M. M. M. (2002). Medidas do comportamento organizacional. Estudos de Psicologia, 7(spe), 11-18.

Tamayo, M. R. \& Trócolli, B. T. (2002). Exaustão emocional: relações com a percepção de suporte organizacional e com as estratégias de coping no trabalho. Estudos de Psicologia, 7(1), 37-46.

Tiedje, L. B. (2004). Process of change in work/home incompatibilities employed mothers. Journal of Social Issues, 60(4), 787-800.

Verschueren, K. \& Marcoen, A. (1999). Representation of self and socioemotional competence in kindergartners: Differential and combined effects of attachment to mother and to father. Child Development, 70(1), 183-201.

Warren, J. A. \& Johnson, E. J. (1995). The impact of workplace support on work-family role strain. Family Relations, 44, 163-169.

Recebido em julho de 2005 Aprovado em agosto de 2006

Sobre as autoras:

Fabiana Cia é psicóloga formada pela Universidade Federal de São Carlos, mestre em Educação Especial pela Universidade Federal de São Carlos e atualmente é doutoranda do Programa de Pós-Graduação em Educação Especial da Universidade Federal de São Carlos/UFSCar, tendo apoio financeiro da FAPESP.

Elizabeth Joan Barham é doutora em Psicologia Social e de Desenvolvimento Aplicado pela University of Guelph/Canadá. Atualmente é professora adjunta do Programa de Pós-Graduação em Educação Especial e do Departamento de Psicologia da Universidade Federal de São Carlos/UFSCar e está cursando pós-doutorado nos Estados Unidos. 